\title{
HIGH-RESOLUTION EMISSION-LINE SPECTROSCOPY OF Be STARS: COMPARISON OF Ha AND WEAK EMISSION LINES
}

Reinhard W. Hanuschik

Astronomisches Institut, Ruhr-Universität Bochum, Postfach 1021 48, D-4630 Bochum 1, FRG

Abstract. For a number of bright southern Be stars, highresolution, high $S / N$ spectroscopy has been performed for $\mathrm{H \alpha}$, Si II $\lambda 6371$ and four Fe II emission lines. With the exception of four stars, both Ha and weak emission line profiles exhibit resolved double-peak structure. Peak separation increases with stellar projected rotational velocity. In many cases, $\mathrm{H} \alpha$ profiles (but not $\mathrm{Fe}$ II lines) show resolved fine-structure (inflections in the flanks) indicating a two-component structure of the disk. Envelope models with arbitrary density and rotational velocity laws have been fitted to the Fe II line profiles with satisfactory results. It is found that the innermost $\mathrm{Ha}$ disk component also emits the Fe II lines and has an average outer radius of $5 r_{*}$; density falls off as $r^{-1.5}$ or more rapidly. The outer disk component emits the faint and narrow secondary component of $H_{\alpha}$ lines with average outer radius $20 r_{\star}$. The envelopes of the four stars with a strong, dominating single peak both in $\mathrm{H} \alpha$ and $\mathrm{Fe}$ II either are dominated by strong radial motions or, more likely, have elliptical disk-like structure.

\section{Measurements}

Al measurements were obtained at the European Southern Observatory at La Silla/Chile using the Coude Echelle Spectrometer with a 1872 pixel reticon fed by the $1.4 \mathrm{~m}$ Coude Auxiliary Telescope during two observing runs in 1985 (Jan. 1 to 5 and Feb. 27 to Mar. 3). For 24 bright southern stars, $H_{\alpha}$ line profiles were obtained and, for 17 of them, also line profiles of Fe II $\lambda \lambda 6384,5317 / 5198,5169$ and 4584 and of Si II $\lambda 6371$. The resolution was $6 \mathrm{~km} / \mathrm{s}$, the $S / \mathrm{N}$ ratio some 100 to 1000. Most profiles are virtually free from instrumental effects which partly affect all other published Be star emission line atlases (e.g., Andrillat \& Fehrenbach, 1982; Dachs et a1., 1986a).

\section{Results}

Both $\mathrm{H \alpha}$ and weak emission line profiles are remarkably smooth. Equivalent widths for Fe II lines are by a factor of $100 \mathrm{smaller}$ than for $\mathrm{Ha}$. From their line profile shapes, all program stars can be divided into two groups, group 1 consisting of the majority of the program stars exhibiting double-peak profiles and group 2 being defined by four stars ( $\mathrm{K} \mathrm{CMa}, \mathrm{HR} 3237, \delta \mathrm{Cen}, \mathrm{x}$ Oph) with a single or strongly dominating peak. Many of the group 1 profiles show more or less symmetric inflections in the flanks of their Ha profiles. This profile shape is most easily interpreted in terms of a two-component structure of the $\mathrm{Ha}$ emitting envelope (as assumed before by Kogure, 1969).

Fe II profiles of group 1 are characterized by double-peak structure with $V \simeq R$, with peak separation $\Delta v_{p}$ approximately twice as large as for the corresponding $H a$ profiles and without double-component structure. $\Delta \mathrm{v}_{\mathrm{p}}$ and FWHM are correlated with stellar projected rotational velocity, $v$ sin $i$, as also observed for $H_{\alpha}$ and already found for $\operatorname{FWHM}(\alpha)$ 
by Andrillat \& Fehrenbach, 1982, and Dachs et a1., 1986a,b. The four stars of group 2 exhibit Fe II lines with a single, asymmetric sharp peak. Si II peaks are at the same position as Fe II peaks, but broader. Variability of these profiles is found to be very small on a two-month timescale.

\section{Conclusions}

The correlation between line width and $v$ sin $i$ observed both for $H_{\alpha}$ and $F e$ II emission lines strongly favours the equatorial-disk model originally devised by Struve (1931). For the average of group 1 stars, the equation

$$
\Delta v_{p} / v \sin i=2 r_{a}^{-j}
$$

given by Huang (1972) for $j=0.5$ (Keplerian rotation) results in a mean outer radius $\left\langle r_{I I}>=20 r_{*}\right.$ for the secondary $\mathrm{H}_{\alpha}$ emitting component and $\left\langle r_{\mathrm{Fe}}\right\rangle=5 r_{*}$ for the $\mathrm{Fe}$ II emitting region (for $j=0.5$ ). Thus Fe II is emitted in the densest, innermost primary disk component whereas the faint outer secondary disk component additionally visible in $H_{\alpha}$ does not produce any detectable amount of Fe II emission.

$\mathrm{B} i$-dimensional, optically thin envelope models with $j=0.5$ or 1 and density law $N \propto r^{-m}$ with $m=0 . .4$ have been calculated following the basic ideas of Huang (1972). The resulting theoretical emission line profiles have been successfully fitted to group $1 \mathrm{Fe}$ II emission line profiles. For seven stars, $\mathrm{m}$ is found to be 2.5; for the remaining profiles, $m$ is smaller. It is concluded that for group 1 stars the Fe II emitting region is slowly expanding.

Emission line profiles of the four group 2 stars are supposedly emitted either in envelopes with strong radial motions or, more likely, in elliptical disks (Huang, 1973). Their complex appearance, however, still prevents at present any safe conclusion about their true nature. Therefore, further high-resolution observations of $\mathrm{Ha}$ and $\mathrm{Fe}$ II emission lines from these stars are highly desirable.

$1986 a, b)$.

Details of this work are published separately (Hanuschik,

Andrillat Y., Fehrenbach Ch.: 1982, Astron. Astrophys. Supp1. 48, 93 Dachs J., Hanuschik R., Kaiser D., Ballereau D., Bouchet P., Kiehling R., Kozok J., Rudolph R., Schlosser W.: 1986a, Astron. Astrophys. Suppl. 63, 87

Dachs J., Hanuschik R., Kaiser D., Rohe D.: 1986b, Astron. Astrophys. 159,276

Hanuschik R.W.: 1986a, Astron. Astrophys. in press

Hanuschik R.W.: 1986b, Astron. Astrophys. submitted

Huang S.-S.: 1972, Astrophys. J. 171, 549

Huang S.-S.: 1973, Astrophys. J. 183, 541

Kogure T.: 1969, Astron. Astrophys. 1, 253

Struve 0.: 1931, Astrophys. J. 73,94 


\section{DISCUSSION FOLLOWING HANUSCHIK}

Buscombe:

The red HeI line showed double emission in $\kappa \mathrm{CMa}$ in 1972 (Bahng and Hendry). Hanuschik:

For $\kappa \mathrm{CMa}$, I also measured the HeI line at $5876 \AA$ and only found absorption which, however, was asymmetric with some spurious trace of emission.

Baade:

The $H \alpha$ profiles of your group 2 stars are in remarkable agreement with the predictions of the Poeckert and Marlborough model (1978, their Figure 3g) for disks seen at an inclination angle of $30^{\circ}$. It appears a nice confirmation of that model that all 4 stars for which you have observed such $\mathrm{H} \alpha$ profiles are seen at such aspect angles (assuming that the stellar vsini roughly measures i).

Hanuschik:

Well, I agree. What you said is especially true for $\chi$ Oph. The Poeckert and Marlborough model you mentioned also has strong radial motions as an important property which then are also common to the 4 group 2 stars and distinguish them from group 1 stars with low vsini (as, e.g. HR 2825).

Granes:

I don't remember, how do you estimate the 20 solar radii size of the $\mathrm{H} \alpha$ envelope? Hanuschik:

I took the average value for the normalized peak separation, i.e. $v_{p} / v s i n i$, for the program stars and then derived $<r_{I I}>=20 R_{*}$ under the assumption of Keplerian rotation, following Huang's equation.

Tarafdar:

The amount of emission on the UV side of Balmer jump depends on the amount of Lyman continuum in the model. Does the observed emission in the Balmer continuum correspond to the Lyman continuum of observed $T_{e f f}, g$ for the star in Kurucz's model?

Hanuschik:

No, Lyman continuum ionization alone cannot provide the observed emission, there must also be some absorption of stellar Balmer continuum photons. 